\title{
Epidemiological, Clinical, Biological, Therapeutic Features and Outcome of Congenital Malaria at the Borgou Regional University Teaching Hospital (CHUD-B) in Benin in 2015
}

\section{Godonou Gratien Sagbo1, Alphonse Noudamadjo2*, Joseph Agossou², Julien Didier Adedemy², Awade Achille Obossou' ${ }^{2}$, Djewessi Saturnin Lokossou²}

${ }^{1}$ Faculty of Health Sciences, University of Abomey-Calavi, Cotonou, Benin

${ }^{2}$ Faculty of Medicine, University of Parakou, Parakou, Benin

Email: godsagbo@yahoo.fr, *alphonse_ndama@yahoo.fr, agossoujoseph@gmail.com, kofadier@yahoo.fr, awadefr2000@yahoo.fr, satudolas88@gmail.com

How to cite this paper: Sagbo, G.G., Noudamadjo, A., Agossou, J., Adédémy, J.D., Obossou, A.A. and Lokossou, S.D. (2017) Epidemiological, Clinical, Biological, Therapeutic Features and Outcome of Congenital Malaria at the Borgou Regional University Teaching Hospital (CHUD-B) in Benin in 2015. Open Journal of Pediatrics, 7 , 263-271.

https://doi.org/10.4236/ojped.2017.74030

Received: October 1, 2017

Accepted: November 18, 2017

Published: November 21, 2017

Copyright (c) 2017 by authors and Scientific Research Publishing Inc. This work is licensed under the Creative Commons Attribution International License (CC BY 4.0).

http://creativecommons.org/licenses/by/4.0/

\section{(c) (i) Open Access}

\begin{abstract}
Background: The prevalence of congenital malaria is getting more and more significant in Sub-Saharan Africa where is a malaria-endemic area. This study aimed to identify the clinical and therapeutic features as well as the outcome of congenital malaria in CHUD-B in 2015. Method: It was a cohort and descriptive study with analytical purpose, carried out in the Mother and Child Department which includes the Gynecology \& Obstetrics and Pediatric Unit of CHUD-B. The study target population consisted of all the infants born in the CHUD-B as well as their mothers. The main variable was the presence of congenital malaria. The independent variables were those related to clinical, therapeutic features and outcome. Results: In the study, among the 300 newborns registered, 57 carried congenital malaria i.e. a prevalence of $19 \% .171$ (57.0\%) of them were males versus 129 (43.0\%) females. Among the 281 mothers involved, 48 presented with malaria in pregnancy i.e. a prevalence of $17.0 \%$. At the end of this research work, the factors associated with congenital malaria were fever in the 3rd quarter and malaria in pregnancy in the mother. Conclusion: Nearly one out of five infants born in the CHUD-B was carrier of congenital malaria and approximately one out six mothers presented with malaria detection during pregnancy. A method based on Polymerase Chain Reaction (PCR) should be implemented during the diagnosis in order to confirm malaria cases among both newborns and mothers.
\end{abstract}




\section{Keywords}

Congenital Malaria, Outcome, Benin

\section{Introduction}

Malaria is one of the leading causes of death among children in Sub-Saharan Africa [1]. Congenital malaria, which is a form due to transplacental passage of parasitized red blood cells, is identified in the infant before the seventh day of life [2] [3]. That disease, rarely mentioned in current practice in the presence of a sick infant, may reach very elevated prevalences in highly malaria-endemic areas in Sub-Saharan Africa, i.e. up to $46 \%$ according to some authors [3]. Abortion, intrauterine fetal death, hypotrophy, prematurity, Congenital Malaria Disease (CMD), increased receptivity to malaria during the first two years of life and maternal death are all consequences of malaria in pregnancy [4]. This research work aimed to study the epidemiological, clinical and therapeutic features as well as the outcome of congenital malaria at CHUD-B in 2015 in order to improve professional practice.

\section{Methods}

This research work was a cohort and descriptive study with analytical purpose, carried out in the Mother and Child Department including the Gynecology \& Obstetric and Pediatric Unit of CHUD-B. The study was extended over the period running from April to July 2015. The study target population consisted of all the infants born at CHUD-B as well as their mothers. Newborns were immediately involved after their birth i.e. within one hour after delivery. The study involved all infants, born alive or not, whose mothers had given their informed consent for inclusion and participation. In cases of intrauterine death, fresh products of conception with gestational age above 22 Weeks of Amenorrhea (WA) or weight above $500 \mathrm{~g}$ were included. The study excluded all HIV-infected parturients and their infants identified through systematic screening on admission after pre-test counseling. The expected sample minimum size was 200 with the prevalence $\mathrm{p}=15.3 \%$ of a study carried out in Lagos (Nigeria) in 2006 [5]. The data were collected using a specific form completed by means of face-to-face interview and review of medical records of mothers and hospitalized newborns. The main variable was presence of congenital malaria. Congenital malaria was diagnosed through Thick Blood Smear (TBS) with May Grunwald Giemsa staining technique according to the World Health Organization (WHO) and positive Rapid Screening Test (RST) on newborn's umbilical cord or peripheral blood [6]. Two forms of congenital malaria were distinguished: congenital malaria infestation (CMI) and congenital malaria disease (CMD). CMI case was defined as newborn having a TBS or a RST positive without any clinical manifestation. CMD case was the newborn with both TBS/RST positive and clinical manifestation. Malaria di- 
agnosis in mothers was performed using the same methods implemented in the laboratory of the Army Teaching Hospital (HIA) of Parakou by senior lab technicians under "double blind" working conditions, in relation to the RST performed in the clinical services. The independent variables were related to the epidemiological, biological, clinical and therapeutic features as well as the outcome. Clinical and parasitological follow-up was carried out. Therapeutic abstention was practiced on the newborns in which CMI was diagnosed. By contrast, all the newborns showing at least one clinical sign of disease with biological evidence of malaria (CMD cases) benefitted from hospitalization, treatment and follow-up for congenital malaria. Malaria was treated using artemisinin-based combination therapy (ACT) by oral route, which is the oral suspension form of "dihydroartemisinin-piperaquine". In case of contraindication to oral administration, specific treatment with intravenous injectable artesunate had been planned for at least 24 hours followed by oral administration of ACT as soon as possible during a total of seven days. All the living newborns carrying trophozoites in peripheral blood were eligible to follow-up. In this group, stillborn infants and those dead at birth were not retained for follow-up. Cases of refusal to participate and newborns whose mothers lived outside the city of Parakou and without phone contact were neither treated nor followed up. For newborns presenting with CMI, clinical and biological follow-up through TBS and RST was implemented at 7 days, 14 days, 21 days and 28 days of life. As regards CMD cases, that same follow-up was performed at 3 days and 7 days of life. The data were entered, analyzed and processed using Epi info software version 7. Word processing and construction of tables were done using application softwares such as Microsoft Word and Excel version 2007.

The measures of central tendency and dispersion (Mode, Average, Standard deviation) served to describe the quantitative variables. As regards description of qualitative variables, ratios and their confidence interval were used. Ratios were compared through Karl Pearson's $\mathrm{Chi}^{2}$ test with significance level of 0.05 .

\section{Ethical and professional considerations}

The CHUD-B Director, the Medical Officer of the Army Teaching Hospital of Parakou and the Heads of the Gynecological \& Obstetric and Pediatric units have authorized by writing the conduct of this study. Mothers' free and informed consent was obtained before any participation. The confidentiality of data was guaranteed.

\section{Results}

\section{Population description}

In this study, 300 newborns were registered. Among them, 171 (57.0\%) were males versus 129 (43.0\%) females, i.e. a sex-ratio of 1.32. 6.3\% (19/300) of included newborns were twins. There were 281 mothers of included newborns. Mothers' mean age was $26 \pm 6.0$ years. The youngest one was 16 years and the oldest 51 years. Mothers were under 30 years of age in $76.6 \%$ of cases $(215 / 281)$. Their ages 
were between 20 and 30 years in $64.1 \%$ of cases (180/281). 78.9\% (45/57) of the mothers of infected infants were under 30 years of age.

\section{Clinical features}

Among the newborns followed up for congenital malaria, eight (08) were asymptomatic. By contrast, 17 newborns had showed signs and were therefore hospitalized. Among those symptoms, jaundice, respiratory distress, fever and anemia were noted respectively in $64.7 \%, 58.8 \%, 52.9 \%$ and $35.3 \%$ of cases. There were 4 cases of severe anemia.

\section{Biological features}

Among the newborns, 24 presented with malaria detected through TBS or RST in peripheral blood as well as in umbilical cord blood i.e. 8.0\% (24/300). The biological evidence of malaria (TBS or RST) was made in 17 newborns (5.7\%) in umbilical cord blood only. Eventually, that evidence was made in 16 newborns (5.3\%) in peripheral blood only.

Table 1 shows the findings of TBS and RST performed on the peripheral blood of newborns and mothers, on mothers' placental blood and on newborns' umbilical cord blood.

Table 1. Distribution of mothers according to findings of TBS and RST performed on newborns and mothers' peripheral blood, mothers' placental blood and newborns' umbilical cord blood at CHUD-B in 2015 .

\begin{tabular}{|c|c|c|c|c|}
\hline & \multicolumn{2}{|c|}{ Mothers } & \multicolumn{2}{|c|}{ Newborns } \\
\hline & \multicolumn{2}{|c|}{ Sizes Ratio (\%) } & \multicolumn{2}{|c|}{ Sizes Ratio (\%) } \\
\hline TBS of peripheral blood & & & & \\
\hline Negative & 244 & 86.8 & 260 & 87.0 \\
\hline Positive & 37 & 13.2 & 40 & 13.0 \\
\hline \multicolumn{5}{|c|}{ RST of peripheral blood } \\
\hline Negative & 241 & 85.8 & 298 & 99.3 \\
\hline Positive & 40 & 14.2 & 2 & 0.7 \\
\hline \multicolumn{5}{|c|}{ TBS of placental blood } \\
\hline Negative & 247 & 87.9 & & \\
\hline Positive & 34 & 12.1 & & \\
\hline \multicolumn{5}{|c|}{ RST of placental blood } \\
\hline Negative & 240 & 85.4 & & \\
\hline Positive & 41 & 14.6 & & \\
\hline \multicolumn{5}{|c|}{ TBS of umbilical cord blood } \\
\hline Negative & \multicolumn{2}{|c|}{-} & 259 & 86.3 \\
\hline Positive & \multicolumn{2}{|c|}{-} & 41 & 13.7 \\
\hline \multicolumn{5}{|c|}{ RST of umbilical cord blood } \\
\hline Negative & \multicolumn{2}{|c|}{-} & 259 & 86.3 \\
\hline Positive & \multicolumn{2}{|c|}{-} & 41 & 13.7 \\
\hline
\end{tabular}


In newborns, parasite density of peripheral blood varied from 200 to $3908 \mathrm{p} / \mu \mathrm{L}$ with an average of $666.0 \pm 78.5 \mathrm{p} / \mu \mathrm{L}$. It also varied from 120 to $16,050 \mathrm{p} / \mu \mathrm{L}$ at the level of the umbilical cord, with average estimated at $1061.7 \pm 61.8 \mathrm{p} / \mu \mathrm{L}$.

\section{Therapeutic features}

In this research work, all the 17 newborns followed up for congenital malaria disease were treated with "dihydroartemisinin-piperaquine" based therapy combination by oral route. Transfusion was practiced in four (04) cases; and phototherapy was performed on eight newborns with jaundice out of the 11 registered. All the 10 cases of respiratory distress were put on oxygen.

\section{Outcome}

As far as parasitology is concerned, all the RST performed at 3 days and 7 days of life in newborns with CMD were negative. However, two TBS performed at 3 days of life were positive; they became negative at 7 days of life. Figure 1 indicates the flow of follow-up of newborns with malaria at CHUD-B in 2015.

\section{Prevalence of congenital and maternal malaria}

Among the 300 newborns involved in the study, 57 carried congenital malaria i.e. a prevalence of $19.0 \%$. Besides, 48 out of the 281 mothers registered in the study presented with malaria in pregnancy i.e. a prevalence of $17.08 \%$.

\section{Associated factors}

Table 2 shows the distribution of newborns according to fever in the $3^{\text {rd }}$ quarter, malaria in pregnancy and parasitemia.

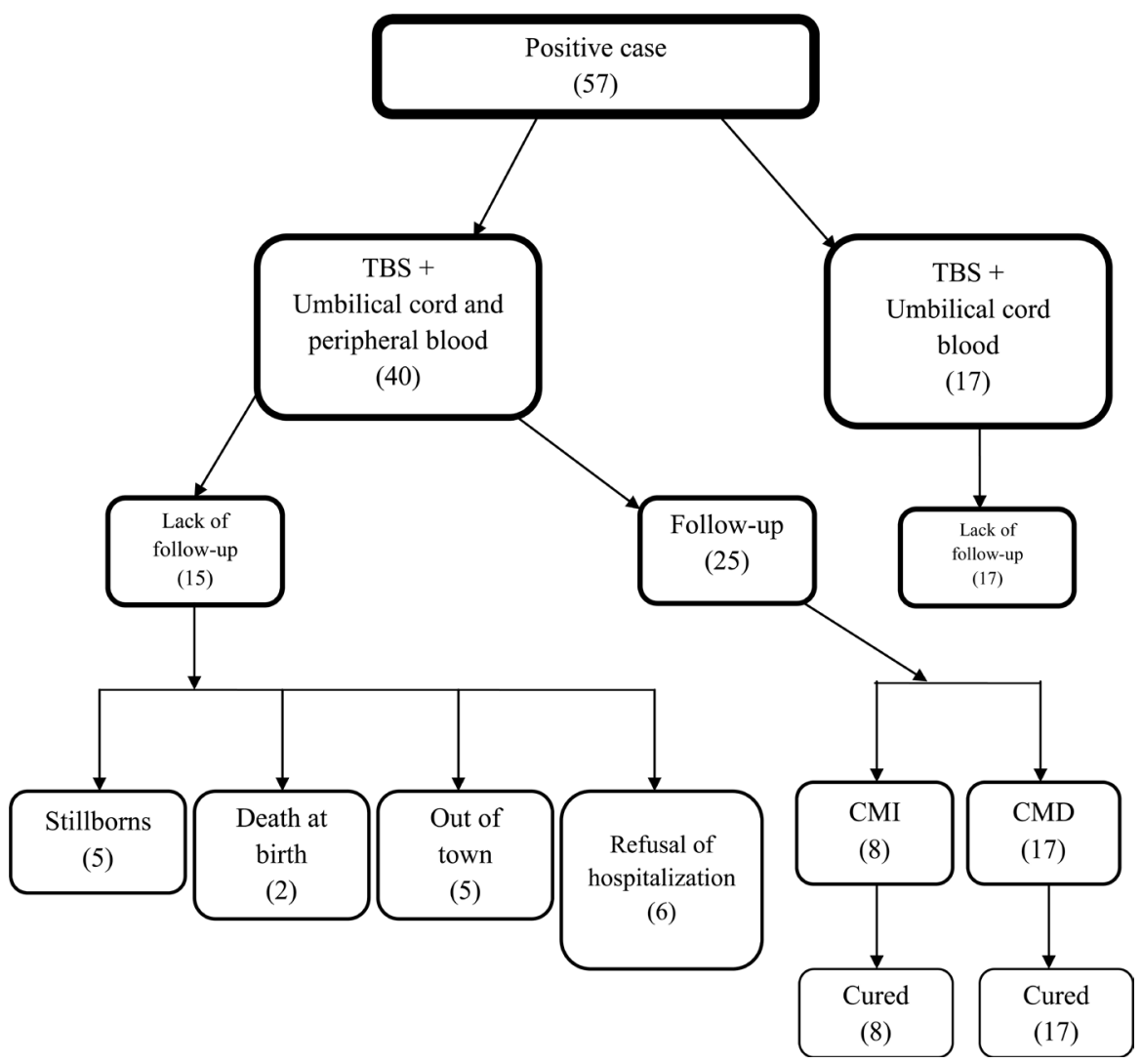

Figure 1. Graph of flow of follow-up of newborns with malaria at CHUD-B in 2015. 
Table 2. Distribution of infants born in the CHUD-B in 2015 according to fever in the $3^{\text {rd }}$ quarter of pregnancy, diagnosed malaria in pregnancy and parasitemia.

\begin{tabular}{ccccccr}
\hline & \multicolumn{7}{c}{ Congenital malaria } \\
\hline & Total & Size & $\%$ & OR & CI & p \\
\hline Fever in the $3^{\text {rd }}$ quarter & & & & & & 0.049 \\
No & 211 & 34 & 16.1 & & 1 & \\
Yes & 89 & 23 & 25.8 & 1.6 & $1.00-2.59$ & \\
\hline Malaria in pregnancy & & & & & & 0.000 \\
Yes & 48 & 33 & 68.8 & & 1 & \\
No & 252 & 24 & 9.5 & 0.14 & $0.09-0.21$ & \\
Peripheral blood parasitemia & & & & & & 0.434 \\
$\geq 1000$ & 15 & 11 & 73.3 & & 1 & \\
$<1000$ & 26 & 17 & 65.4 & 0.89 & $0.74-1.70$ & \\
Placental parasitemia & & & & & & 0.092 \\
$\geq 1000$ & 19 & 17 & 89.5 & & 1 & \\
$<1000$ & 18 & 12 & 66.7 & 0.74 & $0.52-1.07$ &
\end{tabular}

\section{Discussion}

This research work is a study carried out in a hospital setting. This may introduce a bias in the selection through clustering of cases, since malaria in pregnancy may give rise to complications which motivated referrals. Due to time constraints, the study did not cover the whole year. This does not give the opportunity to point out a possible seasonal variability of congenital malaria. However, the prospective character of this study enables to limit missing data during collection. Malaria diagnosis by means of thick blood smear and rapid screening test (RST) was performed by lab technicians under double mind conditions, thus reducing bias in the selection of malaria cases. In addition, this study has the particular merit of addressing a public health issue within the community.

\section{Prevalence of congenital malaria}

This study has demonstrated that one out of five children born in the CHUD-B in 2015 had congenital malaria. Since the objective of the National Policy to Fight Malaria is zero case of malaria in pregnancy and by extension zero case of congenital malaria, that high prevalence (19.0\%) may be due to lack of use of insecticide-treated nets (ITNs) distributed free of charge to all pregnant women. It may be also due to an inadequate follow-up of intermittent preventive treatment during pregnancy (IPTp). Moreover, the prevalence of congenital malaria found out in this research work is lower than the one identified in studies conducted by Chiabi et al. in Cameroon in 2012 (23.79\%) and Kisito et al. in Burkina Faso (24.4\%) in 2013 [7] [8]. Inclusion in latter study was about newborns aged 0 to 7 days. Furthermore, the prevalence of congenital malaria reported in a study involving 120 infants, carried out by Obiajunwa et al. in Nigeria in 2005, was also signifi- 
cantly higher (46.7\%) than the one of this research work [9].

In the study 48 mothers had suffered from malaria during their pregnancy but 57 newborns presented with congenital malaria. That gap or difference may be due to the performance and effectiveness of the two tests done during the diagnosis (TBS and RST). The fact that the ratio of congenital malaria is higher than the one of malaria in mothers indicates that, despite its presence in some mothers, malaria had not been diagnosed with the methods used. Hence, the importance of using other more sensitive methods such as Polymerase Chain Reaction (PCR) for example [10] [11].

\section{Treatment and outcome}

From the therapeutic and outcome point of view, the therapeutic abstention adopted in the case of CMI was conclusive because the 8 cases followed-up had spontaneously negative parasitemia at day 7 of life. This fact has been reported by other authors [12] [13]. However, several studies agree in favor of the treatment of congenital malaria, regardless of the form [11] [14] [15]. Since the number of cases in this study is low, it is desirable to carry out large-scale work before proposing the management of Congenital Malaria.

\section{Associated factors}

This study has pointed out a statistically significant relationship between fever and congenital malaria $(p=0.049)$. This shows that fever remains, even in the pregnant woman, the main sign of malaria. The relationship existing between malaria in pregnancy and congenital malaria is obvious $(\mathrm{p}=0.000)$. Nevertheless, some women, $15.8 \%$ (9/57) of the mothers of newborns exposed to intrauterine Plasmodium falciparum, were not diagnosed as having malaria since TBS and RST performed were negative both on peripheral and placental blood. That situation may be due to parasitemias not detectable by means of microscope according to the techniques used. Ouédraogo et al. have reported in Burkina-Faso two cases of congenital malaria without any evidence of malaria in mother [16]. Parasitemias of maternal placental and peripheral blood were tested but without statistical relationships with onset of congenital malaria. This research work has pointed out that although the ratio of congenital malaria among infants born from mothers with parasitemia higher or equal to $1000 \mathrm{p} / \mu \mathrm{L}$ is higher than the one of infants born from mothers with parasitemia lower than $1000 \mathrm{p} / \mu \mathrm{L}$, the gap was not significant. As a result, the risk for onset of congenital malaria is the same regardless of the value of parasitemia in mother. This is evidence that any malaria in pregnancy control programme should aim to make parasitemia negative and not to reduce it.

\section{Conclusion}

This research work has pointed out that the prevalence of congenital malaria diagnosed by a method combining TBS and RST in immediate neonatal period was higher than the one in mothers using the same methods. As well, the development of congenital malaria does not depend on maternal parasitemia. The differ- 
ence existing between the prevalences of congenital and maternal malaria raises the issue of effectiveness of Thick Blood Smear test and Rapid Screening Test done in hospitals. Moreover, the small number of cases of CMI does not make it possible to conclude on the management of CMI. It would be wiser to conduct a large-scale study taking into account the diagnosis based on PCR and the different therapeutic options.

\section{Acknowledgements}

The authors are especially grateful to:

- The newborns and their mothers;

- The lab Technicians of CHUD-B and Army Teaching Hospital (HIA);

- The workers of the Clinical and Epidemiological Research Unit of the Faculty of Medicine of the University of Parakou.

\section{Conflict of Interest}

No conflict of interest of whatsoever nature is reported by the authors about the conduct of the research work and publication process.

\section{References}

[1] World Health Organization (2013) Proposal Development to the Global Fund to Fight Malaria: WHO Policy Brief. Malaria. Aide-Memoire No.94.

[2] Akaffou Adja, E., Amon-Tanoh Dick, F. and N'Guessan, R. (2009) Epidemiological Study of Malaria in Neonatal Period in the University Teaching Hospital of Yopougnon, Republic of Côte d'Ivoire. Mali Medical, 24, 36.

[3] Monebenimp, F., Chelo, D., Kamo, H. and Obama, M.T. (2013) Congenital Malaria: Diagnostic Difficulties in a Newborn at the University Teaching Hospital of Yaoundé, Cameroon. Health Sciences and Diseases, 14, 1. http://www.hsd-fmsb.org/index.php/hsd/article/view/218/pdf_47

[4] Steketee, R., Nahlen, B., Parise, M. and Menendez, C. (2001) The Burden of Malaria in Pregnancy in Malaria-Endemic Areas. The American Journal of Tropical Medicine and Hygiene, 64, 28-35. https://doi.org/10.4269/ajtmh.2001.64.28

[5] Mukhtar, M., Lesi, F., Iroha, U., Egri-Okwaji, T. and Mafe, G. (2006) Congenital Malaria among Inborn Babies at a Tertiary Centre in Lagos, Nigeria. Journal of Tropical Pediatrics, 52, 19-23. https://doi.org/10.1093/tropej/fmi044

[6] World Health Organization (2014) Basic Techniques for the Microscopic Diagnosis of Malaria. 2nd Edition, WHO, Geneva, 90 p. http://apps.who.int/iris/bitstream/10665/164472/1/9789242547825_fre.pdf

[7] Chiabi, A., Lendem, I., Kobela, M., Mah, E., Tietche, F. and Tchokoteu, P. (2012) Incidence of Congenital Malaria in Two Neonatal Intensive Care Units in Yaounde, Cameroon. Journal of Pediatrics and Child Care, 25, 301-308.

[8] Nagalo, K., Dao, F., Minodier, P., Sawadogo Sanon, H. and Tall, F.H. (2014) Plasmodium Falciparum Congenital Malaria Disease: Epidemiological, Clinical, Biological, Therapeutic Features and Prognosis in Ouagadougou, Burkina Faso. Pan African Medical Journal, 18, 47. https://doi.org/10.11604/pamj.2014.18.47.3614

[9] Obiajunwa, O., Owa, J. and Adeodu, O. (2005) Prevalence of Congenital Malaria in Ile-Ife, Nigeria. Journal of Tropical Pediatrics, 51, 219-222. 
https://academic.oup.com/tropej/article/51/4/219/1670220/Prevalence-of-Congenit al-Malaria-in-Ile-Ife https://doi.org/10.1093/tropej/fmi003

[10] Diouf, F.N., Faye, P.M., Ba, I.D., Ba, A. and Kaimba, L.C. (2015) Prevalence of Plasmodium Falciparum Congenital Malaria Infection in the Regional Hospital of Ziguinchor/Senegal. Revue du CAMES Santé, 3, 70.

[11] Enweronu-Laryea, C.C., Adjei, G.O., Mensah, B., Duah, N. and Quashie, N.B. (2013) Prevalence of Congenital Malaria in High-Risk Ghanaian Newborns: A Cross-Sectional Study. Malaria Journal, 12, 17. http://www.malariajournal.com/content/12/1/17 https://doi.org/10.1186/1475-2875-12-17

[12] Le Hesran, J. (2000) Les particularités du paludisme chez l'enfant. [Features of Malaria in children.] Médecine Tropicale, 60, 92-98.

[13] Cot, M. and Deloron, P. (2003) Paludisme associé à la grossesse: Conséquences et perspectives d'intervention. [Malaria Associated with Pregnancy: Consequences and Prospects for Intervention.] Médecine Tropicale, 63, 369-380.

[14] Xue, L., Zhi-Yong, T., Qiang, F., Xue-Mei, W., Hui, Z. and Jose, A.S. (2012) A Case of Congenital Plasmodium vivax Malaria from a Temperate Region in Central China. Malaria Journal, 11, 182.

[15] Poespoprodjo, J., Fobia, W., Kenangalem, E., Hasanuddin, A., Sugiarto, P., Tjitra, E., et al. (2011) Highly Effective Therapy for Maternal Malaria Associated with a Lower Risk of Vertical Transmission. Journal of Infectious Diseases, 204, 1613-1619. https://doi.org/10.1093/infdis/jir558

[16] Ouédraogo, A., Tiono, A., Amidou, D., Edith, C., Bougouma, C. and Kouaté, T. (2011) Transplacental Transmission of Plasmodium falciparum Malaria Endemic Area of Burkina Faso. Journal of Tropical Medicine, 2012, 1-7. 